\title{
Harmonic components extraction influence on resulting modal parameters of vibrating structures
}

\author{
Deniss Mironovs $^{1}$, Aleksey Mironov ${ }^{2}$, Andris Chate ${ }^{3}$ \\ ${ }^{1,3}$ Institute of Materials and Structures, Riga Technical University, Riga, Latvia \\ ${ }^{2}$ SIA D un D centrs, Riga, Latvia \\ E-mail: 'deniss.mironovs@edu.rtu.lv(corresponding author)
}

\begin{abstract}
It is possible to estimate condition of static structures, like bridges and buildings, by monitoring how their modal parameters (frequency, shape and damping) change in time, based on modal parameters relation to mechanical properties. Operational modal analysis (OMA) provides estimation of modal parameters. OMA basic assumption is that excitation forces are random and their amplitude and frequency nature is similar to white noise. For structures with periodic dynamic excitation, like wind turbines or helicopter blades, this assumption is violated, which makes OMA application complicated and unreliable. There is a challenge of extracting periodic components before modal parameter estimation, which otherwise contaminate signals and mask modal information. This paper considers a signal processing tool for extraction of periodic components from signals aiming to perform OMA and to estimate modal parameters. The tool is based on time synchronous averaging and utilizes tachometer signals. The influence of signal processing technique on modal parameters is studied by comparing these parameters with and without given signal processing tool. The paper also considers the effectiveness of the tool and discusses its possible uses.
\end{abstract}

Keywords: operational modal analysis, structural health monitoring, signal processing, time synchronous averaging.

\section{Introduction}

The field of structural health monitoring is rapidly growing and has its implementation in various fields, including civil engineering. There are several SHM techniques, some of which are based on vibration analysis, particularly on modal analysis (Maia \& Silva, 1997). The latter is aimed at estimating modal parameters, including frequency, mode shape and damping coefficient that are directly related to structural properties, like mass, stiffness and damping. In civil engineering operational modal analysis (OMA) is more practical, because it does not require artificial test excitation of the studied structure, unlike experimental modal analysis (Brincker \& Ventura, 2015).

One descriptive feature of OMA is the assumption that existing natural excitation is essentially a white noise, i.e. frequency spectrum of the exciting force has equal intensity for all frequencies (Mironovs \& Chauhan, 2017). Moreover, it is assumed that excitation is evenly distributed around the system/object. In terms of a building structure these forces can be either natural forces - wind, rain, even earthquake, or man produced sources of excitation - mainly transportation traffic. For specific structures, which are exposed to periodic excitation, e.g. wind turbine tower and blades or helicopter blades, OMA assumption is not met (Mironov, Mironovs, \& Kabashkin, 2018). Periodic excitation can be found from rotating mechanisms, like gearboxes and shafts. Random forces provide even excitation for natural modes, while periodic part creates forced vibrations. The latter should be distinguished from natural modes, which is a challenging task without propper signal analysis. An extraction algorithm can be a valuable tool in dividing recorded signals into random and periodic part. Random part is then used for modal analysis and structural health monitoring, while sole periodic part can be used for diagnostics of rotating machinery (Mironov \& Mironovs, 2018a).

This paper shows application of an extraction algorithm and evaluates its effectiveness and influence on the resulting modal parameters. The effectiveness of the algorithm depends on the quality of modal parameter estimation. A high quality estimation has many modes present in the results, which are uncorrelated between each other, have low complexity and clear modes shapes that correspond to theory of vibrations. 


\section{Modal analysis}

Modal analysis is a system identification technique, used for obtaining parameters of dynamic behaviour of systems. These are modal parameters, mentioned above - frequency, damping and mode shape. This approach is used at design stage of many civil engineering structures, like bridges, tall buildings, wind turbines, as well as for many other structures, including car bodies, aircraft parts, etc (Patel \& Jamadar, 2016). Modal analysis gives information about frequencies at which natural oscillations appear. Engineers exploit this information to avoid these frequency ranges for safety reasons, or even vice versa, use resonances to amplify power of an instrument, for example. Modal analysis can be used for structural health monitoring and damage identification, as well (Zou, Tong, \& Steven, 2000).

\section{Theoretical background of modal analysis}

For a single degree of freedom system the equation of motion is given as

$$
M \ddot{x}(t)+C \dot{x}(t)+K x(t)=p(t),
$$

where: $M$ is the mass matrix, $C$ is the damping matrix, $K$ is the stiffness matrix, $x(t)$ is the response vector and $p(t)$ is the force vector, which in frequency domain is shown as:

$$
\left(-M \omega^{2}+\mathrm{j} \omega C-K\right) X(\omega)=P(\omega)
$$

The Frequency Response Function (FRF) is a relation between response and excitation:

$$
H(\omega)=\frac{X(\omega)}{P(\omega)}=\frac{1}{-M \omega^{2}+\mathrm{j} \omega C-K} .
$$

The FRF mathematical model contains mode shapes, natural frequencies and damping coefficient in the following equation:

$$
H(\omega)=\sum_{r=1}^{N} \frac{A_{r}}{\mathrm{j} \omega-\lambda_{r}}+\frac{A_{r}^{*}}{\mathrm{j} \omega-\lambda_{r}^{*}},
$$

where $A_{r}=Q_{r} \varphi_{r} \varphi_{r}^{T}$. Term $\varphi_{r}$ is a shape for mode $r$ and $Q_{r}$ is the modal scaling factor. The natural frequencies for mode $r$ are obtained from poles in Eq. (4) by

$$
f_{r}=\frac{\operatorname{Im}\left(\lambda_{r}\right)}{2 \pi}=\frac{\omega_{r}}{2 \pi}
$$

and for damping

$$
\zeta_{r}=\frac{-\operatorname{Re}\left(\lambda_{r}\right)}{\left|\lambda_{r}\right|} .
$$

As one can see, modal parameters are dependant on mass, stiffness and damping. Experimental modal analysis is based on Eq. (3) and requires $X(\omega)$ and $P(\omega)$ to be known.

Operational modal analysis

This type of modal analysis is an output-only method, which means that only $X(\omega)$ is measured and $P(\omega)$ remains unknown. It is assumed, though, that excitation is essentially random in nature and evenly distributed across system. An ideal case of such excitation in real world can be rain or traffic induced vibrations, averaged during considerable time. Using this assumption the output is treated like an FRF function for further data processing.

\section{Time synchronous averaging}

This technique is used for obtaining periodic information from a given signal, which is widely used for machinery condition diagnostics (Ha et al., 2016). For structural health monitoring purposes TSA is applicable in determination of periodic component. Time synchronous averaging is a signal processing technique that extracts periodic waveforms from noisy data (Bechhoefer \& Kingsley, 2009). Tacho signal is necessary for order tracking, although, some techniques exist, which allows production of a reference signal for TSA without direct acquisition of tachometer signal (Coats, Sawalhi, \& Randall, 2009). This research does use tacho signal, which gives necessary high precision. A single tone signal in time domain can be presented as

$$
D(t)=D \mathrm{e}^{\mathrm{j} \omega t+\varphi},
$$


where $\omega$ is the frequency of oscillation (e.g. rotor frequency), $t$ is time and $\varphi-$ initial phase of the signal.

A periodic signal is one that combines one or several harmonics of a single tone signal

$$
D_{p}(t)=D_{0}+D_{1} \mathrm{e}^{\mathrm{j} \omega t}+D_{2} \mathrm{e}^{\mathrm{j} 2 \omega t}+D_{3} \mathrm{e}^{\mathrm{j} 3 \omega t}+\ldots+D_{n} \mathrm{e}^{\mathrm{j} n \omega t}=\sum_{k=1}^{K} D_{k} \mathrm{e}^{\mathrm{j} k \omega t},
$$

where each harmonic is a multiple of the fundamental harmonic and $D_{0}$ is the offset, which we assume to be zero for most applications, as signals are highpass filtered during conditioning stage in many applications. The equation for recorded signal with presence of periodic part is expressed as

$$
x(t)=h(t) p(t)+D_{p}(t)
$$

where $h(t)$ is the Impulse Response Function, the FFT inverse of Frequency Response Function $H(\omega)$.

Enhancement is applied for the vibrational signal $x(t)$ while in time domain, utiziling tacho signal. First, indices of samples for each rotation period start are collected. Using these indices time signal $x(t)$ is divided into series of non-equal chunks. These chunks are resampled to the shortest period length and then averaged, which gives the periodic component $D_{p e}(\omega)$ with the fundamental frequency of tacho signal, i.e. rotor. The index $e$ in $D_{p e}(\omega)$ shows that this is experimentally obtained component and can differ from theoretical $D_{p}(\omega)$. Subtracting periodic component from the time signal in time domain (to keep the phase information) gives

$$
x_{p}(t)=x(t)-D_{p e}(t) \text {, }
$$

where $x_{p}(t)$ is a "corrected" response or simply response with extracted harmonic component.

In the following sections modal parameter estimation is performed on $x(t)$ and $x_{p}(t)$ and results are compared.

\section{Experiment}

An experiment was conducted, which was aimed at estimating modal properties of small helicopter composite blades. The experiment included dynamic rotation tests on a special rig.

\section{Measurement system}

The measurement system is integrated into the blade itself and consists of piezo film deformation sensors (Figure 1), thin conducting wires and data acquisition (DAQ) module on the rotating shaft. Sensors are fixed to the blades surface via double sided adhesive tape, which correctly transmits deformations in the frequency region of interest. Sensors and wires are covered with a layer of low density glass fiber mat and epoxy resin. This layer holds the measurement system parts on the blade surface, prevents hazardous impact from fast airflow and centrifugal force, which tends to rip off the sensors and wires during rotation. Epoxy resin is chosen according to the properties of the blades material, so that both layer and the blade pair together successfully.

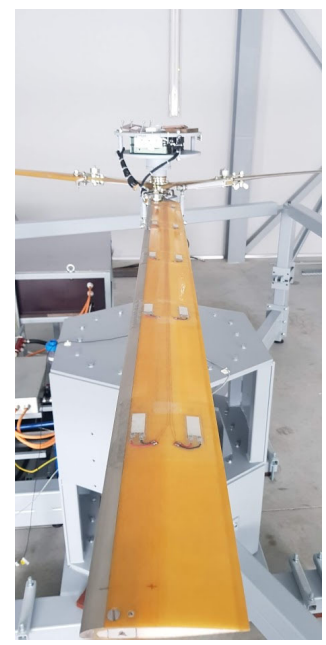

Figure 1. Blade with embedded piezo film sensors and measurement system 
The DAQ is Bruel \& Kjear LAN-XI 12 channel module. It is transmitting acquired signals wireleslly onto PC nearby at the control desk.

There are 5 sections with 2 sensors in each, parallel to the axis of the blade, 10 sensors in total. Additionally a tacho probe is installed on the shaft, which records the speed of rotation, giving impulses as output.

Presented signals are obtained for measurements with 8 degrees angle of attack, which allows the air flow to effectively excite structural vibrations.

\section{Data processing}

Measured signals are 60 seconds long with $4096 \mathrm{~Hz}$ sampling rate. Time synchronous averaging was applied to acquired vibrational signals. Figure 2 shows graphs for the measured signal, that has both periodic and random components ("mixed signal"), periodic signal, accumulated using tacho impulses and the resulting signal ("corrected signal"), which is received by subtracting periodic component from mixed signal. The time domain is shown on the left and frequency domain is shown on the right, using Fast Fourier Transform. The phase alignment of mixed and periodic is very important and is achieved by careful usage of tacho signal.

TSA application allows to determine periodic component, which has its fundamental frequency at 17.1 Hz. Periodic component also has multiple harmonics, which is normal for complex rotary machinery.

The magnitude of the $2^{\text {nd }}$ harmonic at $34.2 \mathrm{~Hz}$ for mixed signal is $57.5 \mathrm{mV}$. Correction reduces this harmonic sixteen times - down to $3.5 \mathrm{mV}$. This scale of reduction is not the same for each harmonic, but it shows the effectiveness of extraction for some. It should be noted, that this type of correction is able to extract periodic component coming from excitation. Algorithm is not able, however, to extract forced responses of the structure itself. This should be kept in mind, while performing modal parameter estimation.

The efficiency of periodic extraction cannot be directly measured. If a spectral magnitude is considered, there is not much of a difference between mixed and corrected signal, but this is not entirely objective, because FFT spectrum is largely dependant on conditioning properties, like time windowing, overlap, sampling rate and others. Additionally, the purpose of extraction is to obtain more correct results of modal parameter estimation. Thus it is more practical to compare mode parameters instead of FFT spectra (or time domain functions for that matter).
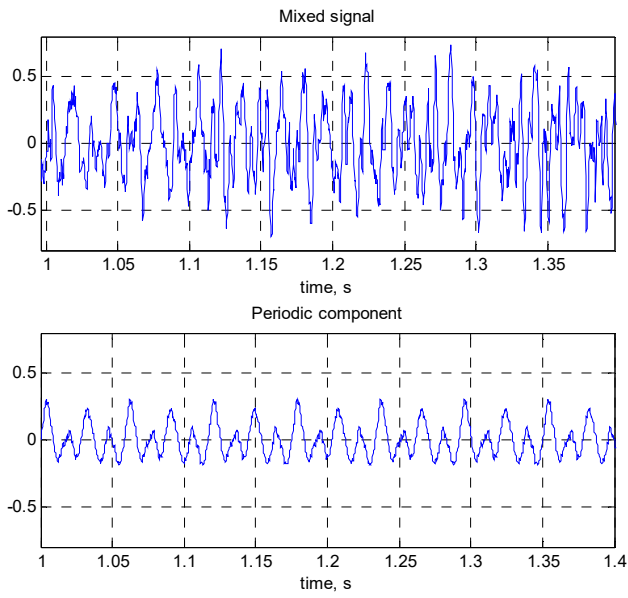

Corrected signal

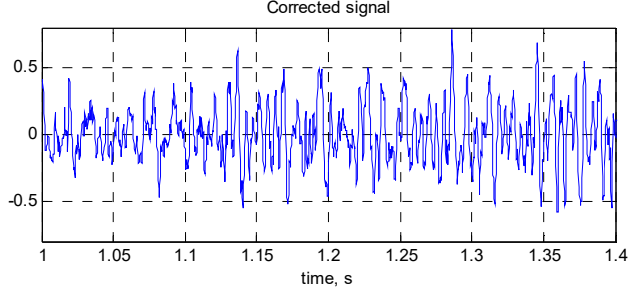

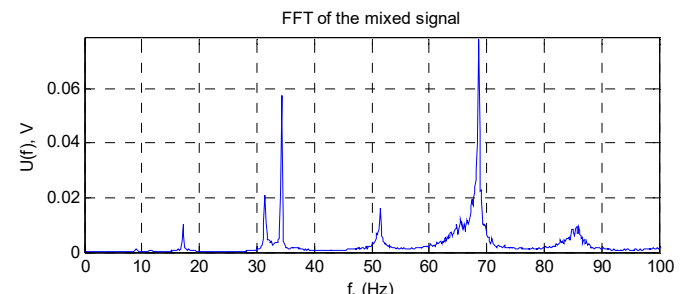
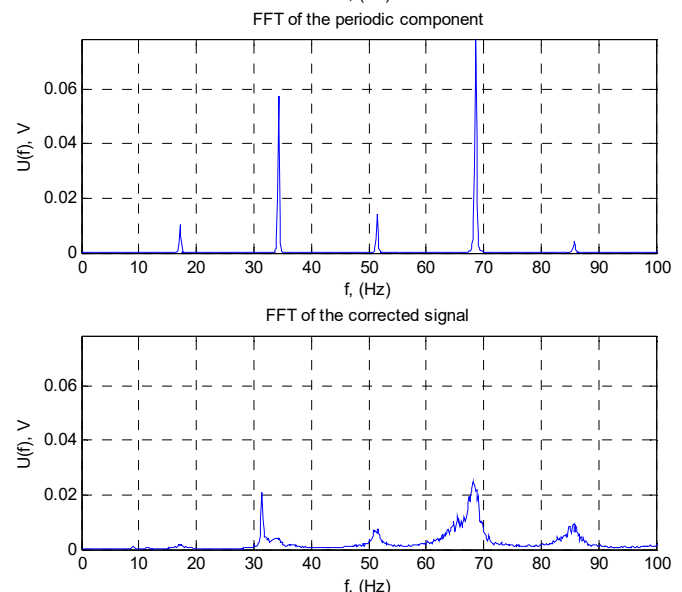

Figure 2. Example of TSA extraction for vibrational signal

\section{Modal parameters estimation}

The procedure of modal parameter estimation was performed using enhanced frequency domain decomposition (EFDD) analysis (Gade, Moller, Herlufsen, \& Hansen, 2005; Chauhan, 2015). EFDD transforms vibrational responses into frequency domain. This technique utilizes conventional peak-picking technique, but in a slightly advanced way. 
The operator chooses a peak, which presumably belongs to a mode. The program then takes the chosen singular vector as a reference vector and compares other singular vectors on both sides of the singular value plot using Modal Assurance Criterion (MAC). This is a correlation coefficient based criterion and is set to be 0.8. Singular vectors, which have MAC value 0.8 or more compared to the reference vector are taken into account. These singular vectors together with the reference vector are averaged out to form a representation of particular mode shape. Averages are weighted by multiplying to corresponding singular values. Corresponding singular values, when plotted, form a characteristic single degree of freedom (SDOF) spectral bell, which is then inverse Fourier transformed into time domain, giving an SDOF correlation function. This, in turn, can be used for natural frequency and damping ratio estimation.

The resulting modal frequency, mode shapes and damping coefficients for 10 estimated modes are shown in Table 1. It has been observed, that the highest change of frequency and damping after processing appears at harmonic frequencies, which is expected, because these frequencies were affected by the extraction algorithm. It is assumed that degradation of harmonic presence allows to better evaluate response of the blade itself, even though it is forced.

As the result of extraction it was possible to estimate two additional modes, one of which lies on harmonic frequency $(34.29 \mathrm{~Hz})$ and another mode $(137.265 \mathrm{~Hz})$ is a high order mode.

In order to quantifically evaluate difference between the same mode shapes before (original) and after (extracted) processing, again MAC is used (Figure 3). Modes can be regarded as similar when MAC is $>0.8$. It is a good indication that same modes pairs (before and after processing) show MAC values close to 1 . That means that processing did not alter modal information in the signals in significant way.

Table 1. Modal frequency and damping of a composite blade. Estimation without and with periodic extraction

\begin{tabular}{|c|c|c|c|c|c|c|c|}
\hline Nr. & \multicolumn{2}{|c|}{$f, \mathrm{~Hz}$} & \multicolumn{2}{|c|}{ damping } & \multicolumn{2}{|c|}{ complexity } & description \\
\hline & original & extracted & original & extracted & original & extracted & \\
\hline 1 & 9.056 & 9.055 & 1.756 & 1.755 & 15.4 & 14.9 & $1^{\text {st }}$ bending $\mathrm{Y}$ axis \\
\hline 2 & 17.143 & 17.267 & 0.337 & 0.864 & 14.3 & 37.4 & forced bending ( $1^{\text {st }}$ rotor harmonic $)$ \\
\hline 3 & 31.44 & 31.44 & 0.234 & 0.235 & 21.2 & 21.3 & $1^{\text {st }}$ bending $\mathrm{Z}$ axis \\
\hline 4 & - & 34.29 & - & 1.058 & & 4.0 & forced bending $\mathrm{Z}$ axis ( $2^{\text {nd }}$ rotor harmonic) \\
\hline 5 & 51.455 & 51.316 & 0.368 & 1.394 & 99.2 & 94.2 & ( $3^{\text {rd }}$ rotor harmonic) \\
\hline 6 & 68.593 & 68.579 & 0.921 & 1.321 & 10.3 & 13.7 & $3^{\text {rd }}$ bending $\mathrm{Z}$ axis ( $4^{\text {th }}$ rotor harmonic) \\
\hline 7 & 84.802 & 84.833 & 0.118 & 0.166 & 70.7 & 66.9 & $2^{\text {nd }}$ bending $\mathrm{Y}$ axis \\
\hline 8 & 118.752 & 118.711 & 1.284 & 1.293 & 0.8 & 0.7 & $4^{\text {th }}$ bending $\mathrm{Z}$ axis \\
\hline 9 & - & 137.265 & - & 0.072 & & 48.2 & \\
\hline 10 & 153.589 & 153.536 & 0.546 & 0.557 & 42.6 & 35.0 & $3^{\text {rd }}$ bending $\mathrm{Y}$ axis \\
\hline
\end{tabular}

Table 2. Mode shapes of a composite blade. Estimation without and with periodic extraction

original extracted


The original mode shape set shows some correlation between shapes, with MAC values $>0.5$, estimated at frequencies falling to different harmonics. This can be an indication that there is some kind of physical connection on these frequencies, which is rotor excitation in this case. Another reason for higher MAC is aliasing of mode shapes for high order modes, which is most probably not the case here. For the pair of modes at 17 and $51 \mathrm{~Hz} \mathrm{MAC} \mathrm{is} 0.523$; for 51 and $68 \mathrm{~Hz}$ MAC is 0.601 . When comparing same pairs of modes between original and extracted sets, MAC value is 0.618 and 0.583 respectively, showing no significant anticipated reduction of MAC values.

Another approach to evaluate difference between before and after processing is to use modal complexity and its measure - Mode Complexity Factor (MCF). A complex mode can appear due to several reasons: non-proportional damping, bad measurements or poor modal parameter estimation and inconsistent data due to e.g. time variant conditions (ARTeMIS, 2017). Complexity close to $0 \%$ means that the mode is real, which is a good sign of a natural mode. Complexity close to $100 \%$ means that the mode is complex. The composite blade is not homogenous and its inner structure changes between sections, so some modes are complex due to non-proportional damping.

As can be observed in Table 1, the harmonic extraction decreases the influences of periodic parts. This results in a decreased complexity for modes $\mathrm{Nr} .5,7$ and 10-5.5\% improvement in average. On the other hand, modes $\mathrm{Nr} .2$ and 6 obtained higher complexity after processing. This might be explained by the fact that mentioned modes are basically a superposition of several modes (most probably of two modes), one of which is a forced harmonic mode and another is a natural one. Decreasing the harmonic part in ensamble brings the natural mode to a higher relative presence. This might lead both to a higher or lower complexity, depending on the initial presence of the harmonic part. Modes that are not in vicinity of harmonics (Nr. 1, 3,8) did not show serious modification of MCF parameter.

\section{Conclusions}

The goal of this study was to experimentally verify the effectiveness of harmonic extraction tool for structural response data, using the example of a composite helicopter blade. Apart from the effectiveness, the overall impact on the dataset was analysed. Modal parameter estimation was performed for the original obtained vibrational response of rotating helicopter blade. A harmonic extraction algorithm was applied on the mentioned dataset, using time synchronous averaging, based on tacho signal. The tool is essentially an ultra precise bandpass combfilter, which attenu-ates magnitudes of the harmonics frequencies. A repetition of modal parameter estimation was done for the processed data and two sets of modal parameters were compared. This included comparison of frequency, damping, mode shapes, MAC and MFC values for mode shapes.

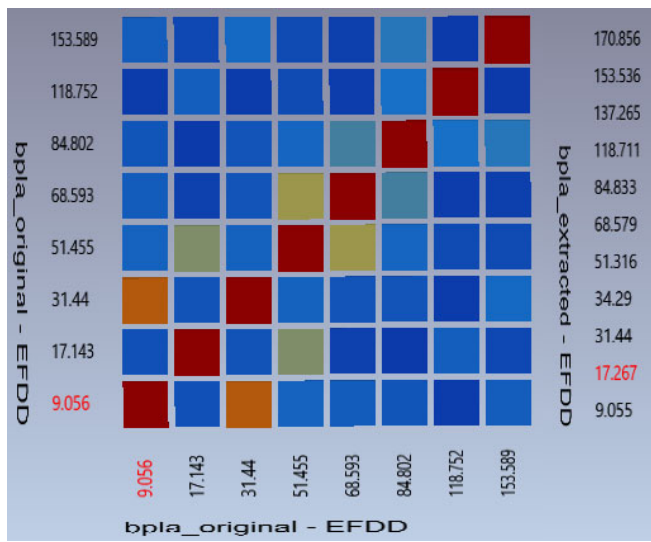

a)

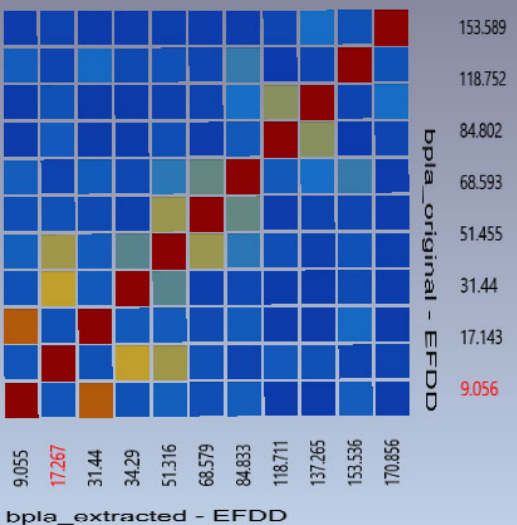

b)

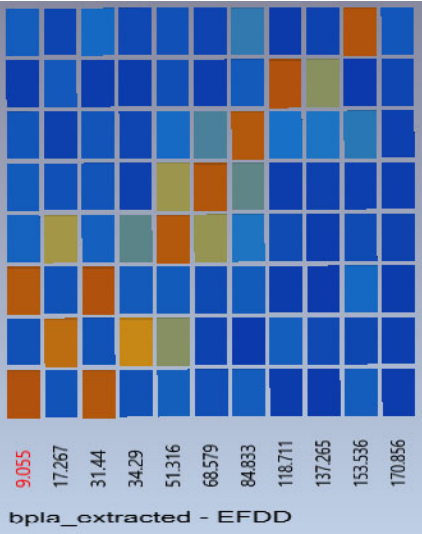

c)

Figure 3. MAC values for mode shapes between original and extracted mode sets: a) autoMAC for original; b) autoMAC for extracted; c) MAC between original vs. extracted

The influence of harmonic components extraction algorithm on modal parameters is rather low, which means also low impact on the response and therefore - low error. The effectiveness of the processing depends on the initial overall presence of harmonics in the given signal. The processing gives possibility to estimate some extra modes, which might be hidden by harmonics. There has been reduced complexity for some modes, although for others it was increased. Nevertheless, the extracted harmonics dataset shows some improvement in the quality of modal parameter estimation. The ability to compare original and extracted versions provides more insight on the structural response and the impact of harmonics in the response, which leads to better understanding of oscillations of the specific object and allows better judgement on the latter. This feature can be used by engineers and researchers for a more precise evaluation of dynamic behaviour of vibration structures under harmonic excitation conditions. 


\section{References}

ARTeMIS. (2017). ARTeMIS Modal Web Help v5.2. Structural Vibration Solutions A/S.

Bechhoefer, E., \& Kingsley, M. (2009). A review of time synchronous average algorithms. In Annual Conference of the Prognostics and Health Management Society. San Diego (CA), USA.

Brincker, R., \& Ventura, C. (2015). Introduction to operational modal analysis. Wiley, New Jersey. https://doi.org/10.1002/9781118535141

Chauhan, S. (2015). Parameter estimation algorithms in operational modal analysis: a review. In $6^{\text {th }}$ International Operational Modal Analysis Conference (IOMAC 2015). Gijon, Spain.

Coats, M. D., Sawalhi, N., \& Randall, R. B. (2009). Extraction of tacho information from a vibration signal for improved synchronous averaging. Proceedings of ACOUSTICS 2009. Adelaide, Australia.

Gade, S., Moller, N., Herlufsen, H., \& Hansen, H. (2005). Frequency domain techniques for operational modal analysis. In Proc $23^{\text {th }}$ International Modal Analysis Conference (IMAC). St. Louis (MO), USA.

Ha, J. M., Youn, B. D., Oh, H., Han, B., Jung, Y., \& Park, J. (2016). Autocorrelation-based time synchronous averaging for condition monitoring of planetary gearboxes in wind turbines. Mechanical Systems and Signal Processing, 70-71, 161-175. https://doi.org/10.1016/j.ymssp.2015.09.040

Maia, N. M. M., \& Silva, J. M. M. (1997). Theoretical and experimental modal analysis. Research Studies Press, Baldock.

Mironov, A., \& Mironovs, D. (2018a). Vibration diagnostics of planetary gearbox of engineering and transport machines based on spatial model application. In: $17^{\text {th }}$ International Scientific Conference "Engineering for Rural Development" : Proceedings. (vol. 17, pp. 1315-1321). Jelgava, Latvia. https://doi.org/10.22616/ERDev2018.17.N108

Mironov, A., Mironovs, D., \& Kabashkin, I. (2018). Advanced structural health monitoring and diagnostics of transport, industrial and energy facilities. I. . Kabashkin et al. (Eds.), Reliability and statistics in transportation and communication. Lecture notes in networks and systems (LLNS, vol. 36, pp. 159-171). https://doi.org/10.1007/978-3-319-74454-4_15

Mironovs, D., \& Chauhan, S. (2017). Modal parameter estimation in multi-patch operational modal analysis: perspectives and approaches. In M. Mains, \& J. Blough (Eds.), Topics in Modal Analysis \& Testing (vol. 10). Conference Proceedings of the Society for Experimental Mechanics Series. Springer, Cham. https://doi.org/10.1007/978-3-319-54810-4_12

Patel, H., \& Jamadar, N. I. (2016). Modal analysis of composite structure. International Journal of Advancement in Engineering Technology, Management and Applied Science, 3(1).

Zou, Y., Tong, L., \& Steven, G. P. (2000). Vibration-based model-dependant damage (delamination) identification and health monitoring for composite structures - a review. Journal of Sound and Vibration, 230(2), 357-378.

https://doi.org/10.1006/jsvi.1999.2624 